\title{
Nanoimprint lithography for planar chiral photonic meta-materials
}

\author{
Yifang Chen ${ }^{\mathrm{a}, *}$, Jiarui Tao ${ }^{\mathrm{a}, \mathrm{b}}$, Xingzhong Zhao ${ }^{\mathrm{b}}$, Zheng Cui ${ }^{\mathrm{a}}$, \\ Alexander S. Schwanecke ${ }^{\mathrm{c}}$, Nikolay I. Zheludev ${ }^{\mathrm{c}}$ \\ a Rutherford Appleton Laboratory, Chilton, Didcot, Oxon OX11 0QX, UK \\ ${ }^{\mathrm{b}}$ Department of Physics, Wuhan University, Wuhan 430072, PR China \\ ${ }^{\mathrm{c}}$ School of Physics and Astronomy, University of Southampton, Highfield, Southampton SO17 1 BJ, UK
}

Available online 11 January 2005

\begin{abstract}
Room temperature nanoimprint lithography has successfully been applied to the fabrication of planar chiral photonic meta-materials. For dielectric chiral structures a single layer of thick HSQ was used while for metallic chiral structures a bi-layer technique using PMMA/hydrogen silsequioxane (HSQ) was applied. The polarization conversion capabilities of planar chiral structures imprinted in dielectric materials have experimentally been observed. This indicates that the developed processes in this work have the prospect of manufacturing planar photonic meta media in high volume at low cost.
\end{abstract}

(c) 2005 Elsevier B.V. All rights reserved.

Keywords: Room temperature nanoimprint lithography; Electron beam lithography; Reactive ion etch; Planar chiral structures; Optical activity; LOR; PMMA; Hydrogen silsequioxane

\section{Introduction}

The recently discovered opportunity to manipulate the polarization state of light in the near- and far-fields using a new breed of meta-materials, so called planar chiral structures (PCS) [1,2], may lead to a whole generation of optical devices and

\footnotetext{
${ }^{*}$ Corresponding author. Tel.: +44 1235 445159; fax: +44 1235446283.

E-mail address: y.chen@rl.ac.uk (Y. Chen).
}

components with potential applications in optoelectronics, communications, and nanotechnology. Nanoimprint lithography, which is typically high speed and relatively low cost, offers a good chance for manufacturing planar chiral structures for optodevices and its components in high volume, especially when only a single layer patterning is needed. In this paper, we report, for the first time that room temperature nanoimprint lithography (RTNIL) delivers high quality metallic and dielectric planar meta-materials with features of nano- 
meter scale. Optical polarization conversion has also been observed on dielectric planar chiral structures fabricated by the RTNIL technique.

\section{Fabrication of sub $100 \mathrm{~nm}$ chiral templates}

In this work, templates with sub-100 nm chiral patterns were fabricated on silicon using electron beam lithography and anisotropic etch. The high resolution E-beam writer VB6-HR was used to pattern the chiral structures in $100 \mathrm{~nm} \mathrm{LOR/}$ $70 \mathrm{~nm}$ PMMA bilayer resists [3] on silicon substrates, followed by a deposition of $10 \mathrm{~nm}$ chrome film and a lift-off process to form the etching mask. A highly anisotropic reactive ion etching process has been developed in a conventional reactive ion etch (RIE) system (System 90 from Oxford Plasma Technology) with a mixture of $\mathrm{CHF}_{3}$ and $\mathrm{SF}_{6}$ gas. The rf power was fixed at $250 \mathrm{~W}$ and the pressure was kept at $30 \mathrm{~m}$ Torr during the etching. Fig. 1 shows the fabricated silicon templates of various chiral patterns with nearly vertical sidewalls. The dimensions are $50 \mathrm{~nm}$ in width and $350-400 \mathrm{~nm}$ in height, respectively. By tuning the ratio of flow rates between the $\mathrm{CHF}_{3}$ and $\mathrm{SF}_{6}$ gases, the verticality of the sidewall can reliably be controlled to meet various needs.

\section{Imprinting at room temperature for dielectric chiral structures}

For the fabrication of dielectric chiral structures, nanoimprint was carried out at room tem- perature on flowable oxide, hydrogen silsequioxane (HSQ). HSQ after curing at temperature beyond its transition temperature $\left(T_{\mathrm{g}}\right)$, turns into silica with refractive index of 1.39. Replicating the chiral patterns on the HSQ layer by RTNIL naturally forms the dielectric chiral structures on silicon substrates.

The HSQ layer was prepared by spin coating a 500-nm FOX24 delivered from Dow Corning Electronics Ltd. on a double polished silicon wafer. It was first baked at $150{ }^{\circ} \mathrm{C}$ for $2 \mathrm{~min}$ on a hot plate and then further baked in oven for $20 \mathrm{~min}$. It was found that a release agent on silicon mould was not necessary when imprinting into HSQ at room temperature. The imprint was undertaken by an in-house made press with a ball joint to keep good uniformity of imprinted depth. In the imprint process a pressure of $33 \mathrm{MPa}$ was kept for $5 \mathrm{~min}$ as the exposure time. The imprinted area is as large as $3 \times 3 \mathrm{~mm}^{2}$. Fig. 2 shows the imprinted chiral structures in HSQ. The imprinted linewidth is around $100 \mathrm{~nm}$. Very well defined chiral patterns have been observed. The characterisation of the optical properties of the imprinted dielectric meta-media will be described in a later section.

\section{Imprinting at room temperature for metallic chiral structures}

Imprint for the fabrication of metallic chiral structures was carried out on double polished silicon substrates at room temperature using an inhouse made press. A bilayer structure with $150 \mathrm{~nm}$ thick PMMA on the bottom and $30 \mathrm{~nm}$
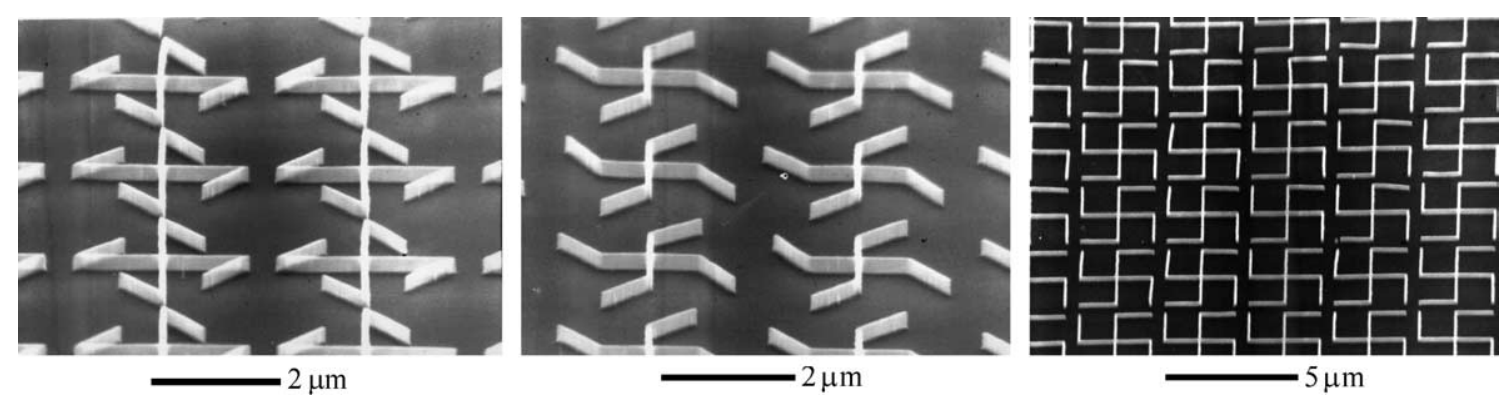

Fig. 1. Micrographs of scanning electron microscope (SEM) for the fabricated templates of various chiral shapes in silicon. The width of the feature is around $50 \mathrm{~nm}$ and the height is $300-400 \mathrm{~nm}$, controlled by dry etch time on Si. 

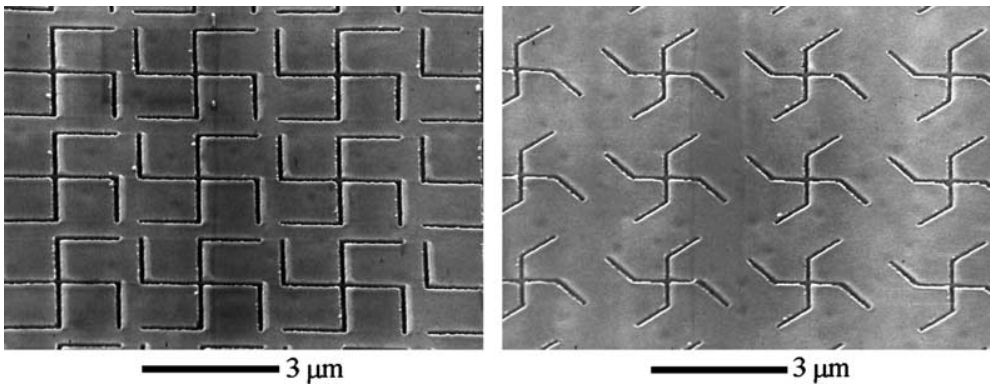

Fig. 2. Micrographs of SEM for the fabricated dielectric planar chiral structures in silica by RTNIL directly on HSQ. 2D optical activity has been observed for such kind of dielectric meta-materials.

HSQ on the top was used [4]. Fig. 3 describes the process flow of the whole fabrication. In this experiment the imprinted depth was controlled by the pressure. This depth is important as it should make sure the templates penetrate the
HSQ layer but do not touch the substrate surface to avoid any damage on both the substrates and the templates. Fig. 4 presents SEM micrographs of templates which had been used in RTNIL for over 50 times. No damage has been observed

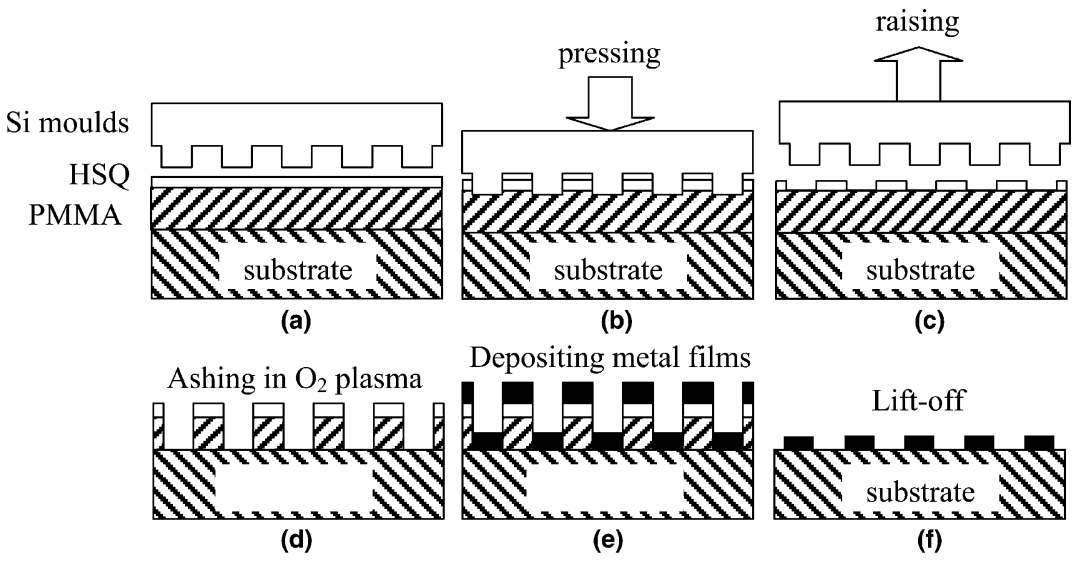

Fig. 3. The process flow for fabricating metallic chiral structures on silica by RTNIL method.

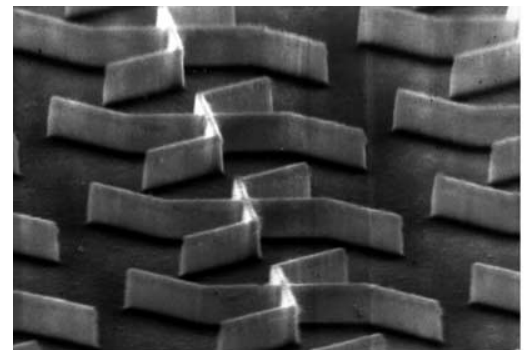

$1.2 \mu \mathrm{m}$

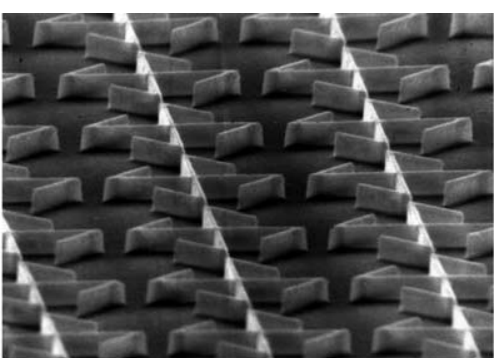

$2 \mu \mathrm{m}$

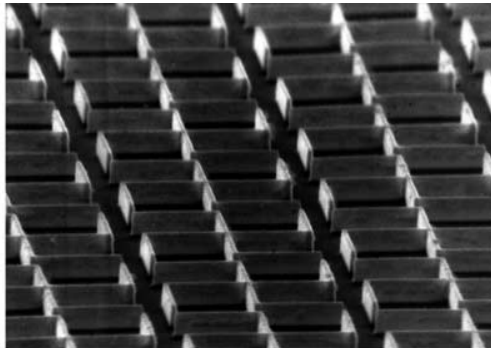

$2 \mu \mathrm{m}$

Fig. 4. SEM micrographs for the used templates after over 50 shots in the RTNIL process. 
throughout the whole chiral area with little amount of resist scum on it, indicating the fabricated templates can be repeatedly used by this process. After imprint, the residual resist, i.e., PMMA was readily removed by a de-scum process in $\mathrm{O}_{2}$ plasma. A metallic film such as PdAu alloy was then deposited by thermal evaporation in an Edward evaporator, followed by lift off in acetone. Fig. 5 presents micrographs of scanning electron microscope (SEM) of fabricated metallic chiral structures in PdAu. However, some broken parts in individual chiral elements were observed. This might be caused by the residual HSQ remaining in the imprinted trenches and was hard to be etched away by $\mathrm{O}_{2}$ plasma. One possible solution is to increase the thickness of PMMA at the bottom to allow deeper penetration of templates. Reducing the thickness of HSQ is another alternative. In this case, presumably less HSQ would remain at the bottom of the trenches so that the residual resist (PMMA only) should be more easily cleared.

\section{Observation of $2 \mathrm{D}$ optical activity in planar dielectric meta-materials by RTNIL}

Natural media with 3D chiral substructures have long been known to exhibit optical activity, i.e., particularly to affect the polarization state of light. An analogues effect has recently been found in planar structures. Planar structures are called chiral if they fail to be their own mirror image, also know as enantiomer [1]. Relating to how 'far' away an object is from having a mirror symmetry in the plane levels of chirality can be defined. Crosses are inherently achiral structures, but motivate a simple class of chiral structures through bend of their arms: gammadions. They have been chosen as templates to investigate the continuous change of chirality associated with their bending angle in relation to its impact on the polarization state of interacting light. This effect can be expected to be most pronounced for structure sizes comparable to the wavelength of probing light. RTNIL fabrication is dedicated to dimensions equivalent and below the wavelengths for visible light while providing a consistent combination of precision and complexity over the large areas needed for various optical applications.

Observations of optical manifestations of planar chirality in dielectric meta-materials fabricated by RTNIL on HSQ layer have been performed, for the first time, in reflective mode. Fig. 6 is a schematic diagram of the experimental setup. A white light halogen source and a narrow-bandwidth filter $(\lambda=550 \mathrm{~nm})$ formed quasi-monochromatic light, which was linearly polarized before incident on the structures. Using a $40 \times$ microscope objective and a 6.3 megapixel low noise CMOS CCD camera, polarization sensitive microscopy could be recorded. Fig. 7 contains photographic images taken under four different orientations of the incident electromagnetic field, revealing the polarization conversion capabilities of the imprinted planar chiral nanostructures. The sample was imaged through a "crossed" linear analyzer, resulting in a dark field for the unstructured and isotropic
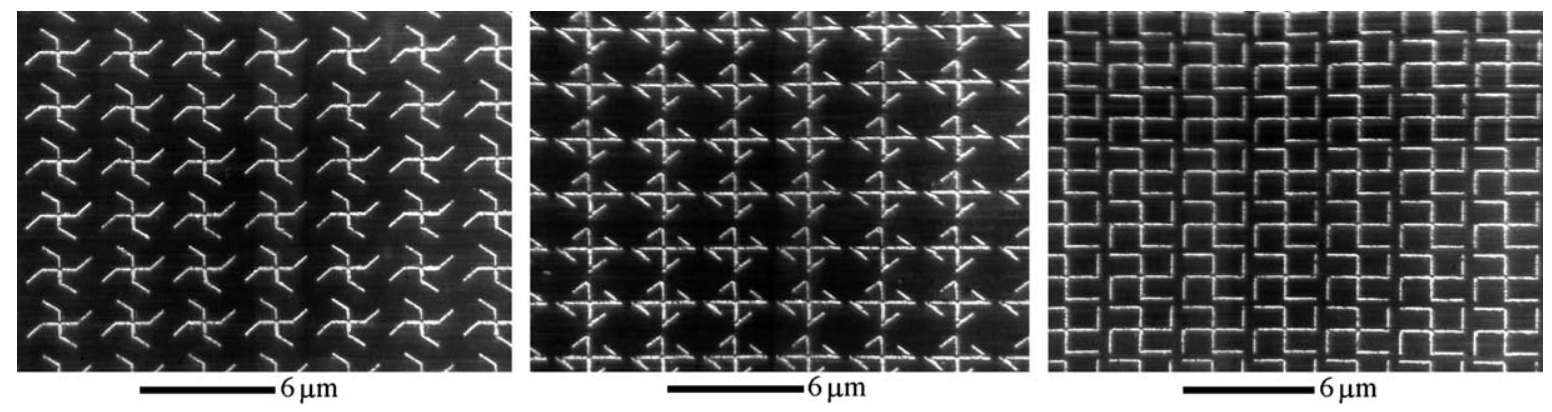

Fig. 5. SEM micrographs of the metallic planar chiral structures fabricated by RTNIL followed by metallization and lift off processes. The metal is a $50-\mathrm{nm}$ thick PdAu film. 


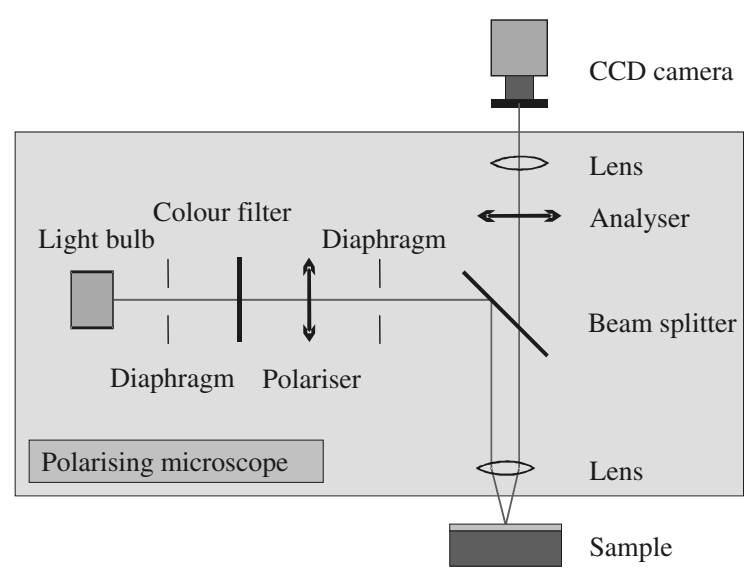

Fig. 6. A schematic diagram of the experimental setup to observe the optical activity of the fabricated planar dielectric meta-materials. For a detailed description, see text.

parts of the dielectric layer. The quasi-monochromatic contrast enhanced observations highlight the regions of optical activity, as shown in
Fig. 7(a). Variation of the mutual arrangement of the incident linear polarization and the gammadion array demonstrate the angular dependency of the polarization conversion process, as shown in Figs. 7(b)-(d). While the sub-wavelength structure of individual elements cannot be resolved, their external branches bear resemblance to the evolving line structures in the images.

Throughout the work, the gammadion shape (a cross-plus four bending arms) as shown in Figs. 2, 4 and 5, was selected. This is mainly due to two reasons. One is that such kind of gammadion shape is the simplest planar chiral structure. The other is that optical activity of such kind of planar chiral structures has already been observed [1,2], which were manufactured by electron beam lithography. This work is to demonstrate that such a chirality also exists in those fabricated by nanoimprint lithography, which is the main focus of this paper. These are first time
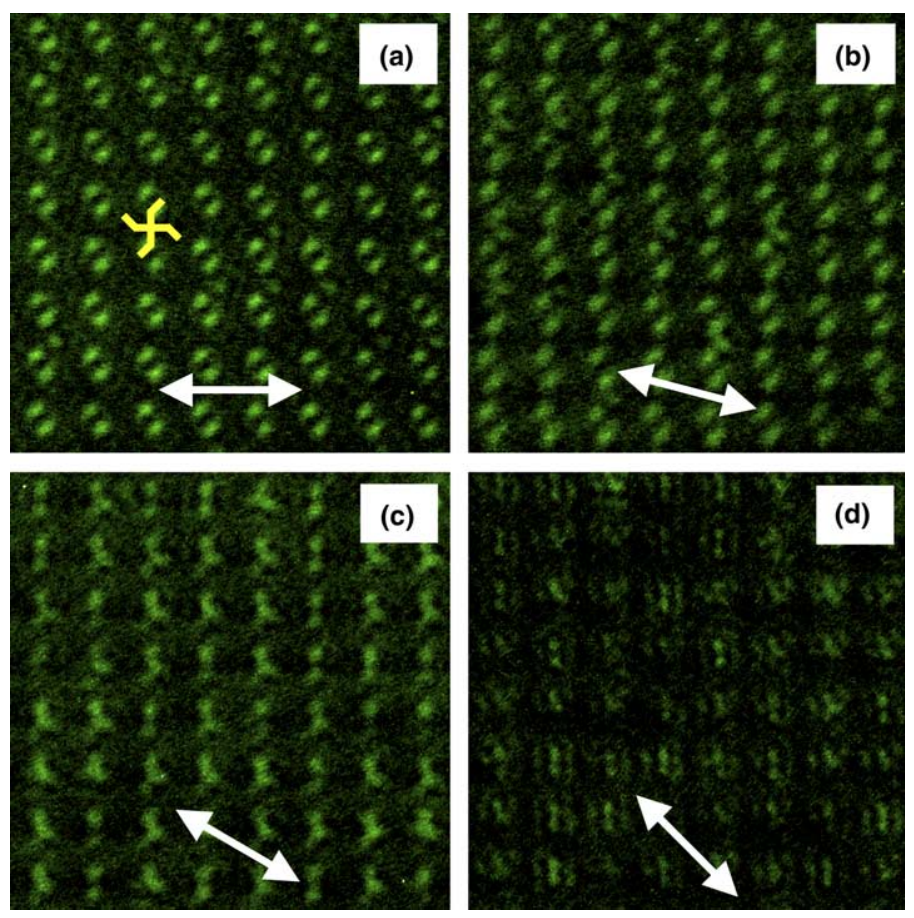

Fig. 7. Photos taken by a CMOS CCD camera showing the cross-polarized observations of polarization conversion capabilities of the imprinted dielectric planar chiral nanostructures with incident linear polarized light. Its E-field is indicated by double ended arrows. (a) The most pronounced polarization conversion is seen for the horizontal configuration - a sketch of a single gammadion has been added. (b-d) Other incident polarization azimuths result into an accentuation of other parts of the planar chiral structure with fewer overall conversion. 
observations of optical activity in dielectric chiral structures fabricated by RTNIL, indicating that the developed production process is applicable for the manufacture of micro- and nanosized planar meta-materials.

\section{Conclusion}

We have reported, for the first time, to fabricate planar chiral photonic meta-materials using room temperature nanoimprint lithography for the study and application of novel polarization effects. Both dielectric and metallic meta-materials with feature sizes from micrometric scale down to sub $100 \mathrm{~nm}$ have successfully been fabricated. Optical activity of dielectric chiral structures has subsequently been observed. However, the process for metallic chiral structures still needs to be improved. Nevertheless, the RTNIL process developed in this work has shown to be a promising candidate for manufacturing novel optic devices and components with planar chiral structures at low cost and high throughput.

\section{Acknowledgement}

The work was partially supported by the European Project "BMR-TFNC" (505282).

\section{References}

[1] A. Papakostas, A. Potts, D.M. Bagnall, S.L. Prosvirnin, H.J. Coles, N.I. Zheludev, Phys. Rev. Lett. 90 (2003) 107404.

[2] A. Potts, A. Papakostas, D.M. Bagnall, N.I. Zheludev, Micro. Nano Eng. 73-74 (2003) 367.

[3] Y. Chen, Kaiwu Peng, Zheng Cui, Micro. Nano Eng. 73-74 (2003) 278.

[4] Jiarui Tao, Yifang Chen, Xingzhong Zhao and Zheng Cui, in: The International Conference of Micro- and Nano Engineering, Rotterdam, The Netherlands, 2005 (this issue). 